\title{
The Advantages and Disadvantages of Transgastric Natural Orifice Transluminal Endoscopic Gallbladder-Preserving Surgery Compared with Traditional Operation and its Clinical Application
}

\author{
Xian-bin Zhou ${ }^{1 \#}$, Min Quan ${ }^{2 \#, ~ K u a i ~ M a ~}{ }^{3}$, Li-ping $\mathrm{Ye}^{1}$, Xin-li Mao ${ }^{1}$, Jian-fen $\mathrm{Wu}^{1}$, Shi-wen $\mathrm{Xu}^{4}$, Cai \\ yue $^{1}$, Bin-bin Gu${ }^{1}$, Yu-Zhang ${ }^{1}$, Li Wang ${ }^{5}$, Jin-bang Peng ${ }^{1}$, Chi Liu ${ }^{2 *}$ and Shao-wei Li ${ }^{1 *}$ \\ ${ }^{1}$ Department of Gastroenterology, Taizhou Hospital of Zhejiang Province affiliated to Wenzhou Medical University, LinHai, \\ Zhejiang, China
}

${ }^{2}$ Reproductive \& Women-Children Hospital, School of Medical and Life Sciences, Chengdu University of Traditional Chinese Medicine, Chengdu, China

${ }^{3}$ Division of Transplantation Immunology, National Research Institute for Child Health and Development, Tokyo, Japan

${ }^{4}$ Wenzhou Medical University, Wenzhou, Zhejiang, China

${ }^{5}$ College of Basic Medicine, Inner Mongolia Medical University, Hohhot, Inner Mongolia, China

\#These authors contributed equally to this work

*Corresponding author: Shao-wei Li,Department of Gastroenterology, Taizhou Hospital of Zhejiang Province affiliated to Wenzhou Medical University, LinHai, Zhejiang, China

Chi Liu, Reproductive \& Women-Children Hospital, School of Medical and Life Sciences, Chengdu University of Traditional

Chinese Medicine, Chengdu, China

\section{ARTICLE INFO}

Received:

Published: September 14, 2020

Citation: Xian-bin Zhou, Min Quan, Kuai $\mathrm{Ma}$, Li-ping Ye, Xin-li Mao, Jian-fen Wu, Shi-wen Xu, Cai yue, Bin-bin Gu, Yu-Zhang, Li Wang, Jin-bang Peng, Chi Liu, Shao-wei Li. The Advantages and Disadvantages of Transgastric Natural Orifice Transluminal Endoscopic Gallbladder-Preserving Surgery Compared with Traditional Operation and its Clinical Application. Biomed J Sci \& Tech Res 30(2)-2020. BJSTR. MS.ID.004936.

Keywords: Notes; Transgastric Natural Orifice Endoscopic Surgery; Cholelithiasis; Gallbladder Preservation

\section{ABSTRACT}

In recent years, the incidence rate of gallstones has been increasing under the multiple influences of environment, heredity and diet. In the modern science and technology developed, treatment effect requirements are higher and higher. At present, no scar, less trauma, fast recovery of the NOTES surgery is more popular. Based on the understanding of NOTES, after searching the literature about gallbladder disease or clinical application, combined with related cases, this paper summarized the treatment measures, specific advantages and application cases of transgastric gallbladder conserving surgery. It has the advantages of no scar, less pain, faster recovery, fewer complications, shorter hospital stay and a series of important functions of gallbladder. At present, the key point of treatment is to select the most appropriate surgical method according to individual differences, physiological and genetic characteristics, disease characteristics, personal willingness and tolerance.

Abbreviations: NOTES:Natural Orifice Translumenal Endoscopic Surgery; APC: Endoscopic Argon Ion BeamCoagulation; OTSC: Over-The-Scope-Clip System;LC:Laparoscopic Cholecystectomy; ESD: Endoscopic Submucosal Dissection; SCC: Esophageal Squamous Cell Carcinoma; PSC: Primary Sclerosing Cholangitis; DVT: Deep Venous Thrombosis; PE: PulmonaryEmbolism Competition

\section{Introduction}

Cholelithiasis refers to the gallstone that occurs in the gallbladder. The main component is cholesterol. The cholesterol in bile is dissolved in water in the form of bile salt phospholipid microcapsules and bile salts. The incidence rate of cholelithiasis 
has increased from $10 \%$ to $20 \%$ in recent years [1]. Transgastric approach of gallbladder preservation surgery refers to With the help of transparent cap, a full-thickness endoscopic incision was made in the posterior antrum near the lesser curvature of the stomach and the anterior wall of the lower part of the gastric body with hook knife and its knife [2]. After full-thickness resection, the greater omentum in the abdominal cavity was separated layer by layer, and the passage between stomach and abdominal cavity was opened, Then the gallbladder was found in the abdominal cavity, and a small incision was made at the junction of gallbladder with hook knife [2]. With the same tool, the window was enlarged to allow the endoscope to enter the gallbladder, and the gallbladder was pulled by the clip with inner ring, so that the endoscope could enter the gallbladder easily. The stone was removed with stone basket or snare traction, APC and hot biopsy forceps to remove gallbladder polyps [2,3].

After the operation, the local gallbladder opening was closed with metal clip, and the gastric wall defect was closed with OTSC (ultra-mirror clip) combined with metal clip [3]. Traditional open surgery or laparoscopic cholecystectomy to achieve the purpose of treatment of stones or polyps, although the effect is good, but the gallbladder as an organ with a variety of physiological functions, such as absorption, secretion, immunity, plays an important role in human metabolism. Many studies have confirmed that cholecystectomy is associated with the incidence rate of colorectal cancer, choledocholithiasis, digestive function impairment, bile duct injury, immune defense function, chronic liver injury, metabolic abnormalities, hepatic steatosis and metabolic syndrome $[3,4]$. Patients after cholecystectomy are prone to abdominal pain, diarrhea, jaundice, bleeding, reflux gastritis, reflux esophagitis, bile leakage, intestinal obstruction, peritonitis and other complications $[5,6]$.

Therefore, it is of great value to treat gallbladder diseases with NOTES. Notes refers to a series of surgical interventions through some natural lumen, such as stomach, colon, rectum, vagina, oral cavity, urethra, etc. into the abdominal cavity to reach different direct target organs or distant target organs, and then carry out a series of surgical intervention [7-9]. However, notes still has some defects. How to avoid the complications of exogenous infection, avoid the anastomotic leakage caused by poor closure, overcome the disorder of intraperitoneal spatial orientation, and select the patients who are mainly suitable for notes need to be explored in clinical practice [2]. In this review, we aim to outline the advantages and disadvantages of choledocholithotomy under gastroscope compared with traditional surgery and related indications and contraindications.

\section{Characteristics and Treatment Options of Gallstones}

Cholelithiasis is a mass of the gallbladder or biliary tract caused by the abnormal elevation of cholesterol or bilirubin (the decomposition product of heme) in the bile [10]. The incidence of cholelithiasis increased with age, the prevalence of cholelithiasis in female was higher than that in male, and the genetic tendency was obvious [10]. Due to the type, location, characteristics, accompanying symptoms and individual characteristics of gallstones, there are many methods to treat gallstones. From traditional open surgery to later laparoscopic surgery, and then to the NOTES operation. Notes is the third generation of operation after open surgery and laparoscopic surgery. It refers to the operation mode of various diagnosis and surgical operation through hollow viscera or natural opening (such as vaginal vault, stomach, etc.) into peritoneal cavity [11]. As there is no abdominal incision in notes, there are no abdominal wall complications, which can achieve good cosmetic effect and reduce anesthesia requirements [12].

The treatment of gallstone and polyp is more and more advanced. Compared with traditional laparotomy, NOTES has the advantages of further reducing trauma, abdominal wall or chest wall trauma, postoperative pain, postoperative recovery time, postoperative complications, wound infection, and other advantages, and there are long-term problems such as hernia reduction, postoperative inflammatory response is not obvious, less physical discomfort, almost no visible scars [13-15]. Therefore, NOTES is a good choice to treat gallbladder disease.

\section{Clinical Application of Notes}

NOTES can be roughly divided into two categories: remote NOTES and short NOTES. Remote NOTES refers to the process of getting from one healthy visceral organ to another distant organ; Short notes refers to the procedure of directly entering the target organ without interfering with another organ (e.g., oral endoscopic myotomy [poem]) [16]. This article describes the stomach through the road to protect the gallbladder stone surgery is attributed to the former. Short range notes are widely used at present, Endoscopic submucosal dissection (ESD) has achieved significant initial results, including endoscopic full-thickness resection of gastrointestinal submucosal tumors, oral endoscopic myotomy and submucosal tunnel endoscopic resection $[17,18]$. And oral endoscopic myotomy (POME) for children with achalasia of cardia [19]. In addition, endoscopic submucosal dissection (ESD) is becoming the preferred treatment for early esophageal squamous cell carcinoma (SCC), including highly atypical hyperplasia and superficial esophageal cancer [20].

For long-term notes, many departments have already used them. A series of experiments have proved the feasibility of adrenalectomy, uterus surgery and gallbladder surgery through natural orifice surgery in general surgery, urology and Gynecology, but the clinical application is very limited [11,21]. For patients with intra-abdominal emergency, when a series of diseases such as ulcer perforation and infectious pancreatic necrosis caused by interruption of blood supply (bleeding, ischemia) or normal 
gastrointestinal tract (obstruction, perforation or leakage) need emergency intervention, the application value of notes is more important. Compared with other surgical methods, notes not only reduces intra-abdominal invasion, but also improves the prognosis [22]. In addition, notes also has relevant clinical application in weight loss surgery [23].

From open surgery to laparoscopic surgery, laparoscopic cholecystectomy (LC) is now considered as the standard for the treatment of cholelithiasis. The acceptance of LC in the world is mainly due to the quick recovery, good cosmetic effect, short hospital stay and early return to work [24]. However, the gallbladder is an important organ of human metabolism, there will be a series of sequelae after resection. Studies have shown that cholecystectomy is associated with an increased risk of prostate cancer in patients with gallstones, and the risk also increases with the extension of follow-up time. Cholecystectomy may not be the appropriate choice for patients with high-risk prostate cancer [25]. And people with a history of cholecystectomy are more likely to develop liver cancer [26].

Therefore, with many complications after cholecystectomy and people's understanding of the important functions of the gallbladder, gallbladder conserving surgery began to develop. At present, notes can be carried out through stomach, rectum and esophagus, among which transvaginal is the most common way. Vaginal subtotal hysterectomy and transvaginal appendectomy have been reported. Transvaginal subtotal hysterectomy has been proved to be feasible and safe by related studies, and it has the advantages of less pain, no scar, and rapid recovery $[9,27,28]$. However, there are few studies on gastric approach. In 2004, Kalloo and Colleagues were examined by gastroperitoneoscopy for the first time in a pig model [29]. Next, Rao and reddy21 demonstrate the first human gastric NOTES appendectomy [29]. Jacques Marescaux performed the first gastric cholecystectomy in humans [29]. These studies have opened the way for minimally invasive surgery via gastric approach. In addition, compared with the rectal approach, the transabdominal approach not only has a shorter route, but also saves time and does not need to clean the intestine [3]. Therefore, transgastric approach is the first choice for some researchers because it has no gender specificity, low risk of infection and strong healing ability of gastric wall [30].

\section{Indications of Gallbladder Preservation}

At present, there is no complete consensus on the indication and application scope of transgastric notes choledocholithotomy. The most common indication of open cholecystectomy was severe cholecystitis (72.1\%), followed by cirrhosis, cholelithiasis and portal hypertension (18.2\%), empyema or gallbladder perforation (6.1\%) [31]. Therefore, choledocholithotomy is not recommended for patients with the above disease characteristics. Laparoscopic cholecystectomy is more suitable for patients with primary sclerosing cholangitis (PSC) due to the increased risk of malignant transformation; patients with venous thrombosis risk are more suitable for cholecystectomy rather than gallbladder conserving surgery, because relevant studies show that gallstones will increase the risk of venous thrombosis, including DVT or PE, and the risk of DVT and PE in patients with gallstone after cholecystectomy is reduced [32].

Most guidelines recommend that cholecystectomy be considered for polyps $10 \mathrm{~mm}$ or larger and smaller but growing polyps [33]. For gallbladder polyps or stones which are not included in the above scope, transgastric choledocholithotomy (or polyp) can be performed. For patients with prostate cancer, according to previous research results, cholecystectomy may not be an appropriate choice, so we can think that such patients may be more suitable for the treatment of notes gallstone. Although notes have the advantages of less trauma and faster recovery than traditional surgery, there are still some problems. Among them, disinfection method, closure technique and operation experience are the most important problems.

As for the choice of disinfection methods for gastric notes, intravenous antibiotics as a traditional disinfection method still has exact clinical significance, but whether single intravenous antibiotics can achieve the purpose of controlling infection remains to be further confirmed [34,35], The local mucosal disinfectants mainly include antibiotic suspension, dichlorobenzidine hexane and povidone iodine. There are also extensive reports on their application. The disinfection effect and safety are worthy of partial affirmation. However, compared with the intravenous application of antibiotics, whether the efficacy of local disinfection is better is still worth discussing.

\section{Disadvantages and Future Development of Transgastric Natural Orifice Endoscopic Surgery}

For the technical problems of gastric cavity closure, because the complications of vaginal wall closure are smaller than those of digestive tract closure failure, so for the choice of notes abdominal approach, vaginal approach is more preferred than gastric approach [29]. And through the vagina surgery case and research is also more, in this aspect also has the sufficient experience. However, transvaginal approach has the disadvantage of gender specificity. Therefore, transgastric approach for natural cavity oral surgery has a good development prospect. However, at present, transgastric notes surgery still faces the challenge of the feasibility and safety of access closure [30]. The types of closure techniques reported include traditional titanium clip, OTSC clip, endoscopic suture system, endoscopic stapler, diaphragm occluder, absorbable biological plug, ndoplicator, g-prox, t-tags and so on. However, it is not clear which closure technology has the advantages. At present, it is widely accepted that OTSC combined with metal clip is used to close the local gallbladder opening and gastric wall defect after 
operation [3].

OTSCs and greater omental plasty are the most promising techniques for safe and effective closure of notes gastric pathway [36]. Whether there will be postoperative gastric leakage and local peritoneal infection need further clinical research to determine the most appropriate use of guidelines [30]. However, the cost, training, operation time and tools seem to limit their application in common operations such as cholecystectomy and appendectomy [37]. With the development of science and technology, robot natural oral mirror surgery has entered into innovation. According to relevant research, robot notes hysterectomy has the advantages of feasibility, safety, no scar, no pneumoperitoneum, and the ability to improve human efficacy and range of motion [38]. In the surgical treatment of benign uterine diseases, it is better in fine anatomy, hemostasis and suture, and can replace notes assisted vaginal hysterectomy or traditional robotic laparoscopic single site hysterectomy. However, due to its shortcomings and safety inflammation, it has not been widely used in clinical practice [38]. Through the stomach natural oral mirror surgery can also be studied robot operation, to achieve more precise surgical standards. This may also be a direction for future development.

\section{Conclusion}

There are many ways to treat gallstones and polyps. At present, laparoscopic cholecystectomy is the most common treatment, and the treatment of gallbladder preservation by laparoscopy is also recorded. The focus of this paper is on the gallbladder preservation therapy of NOTES surgery. At present, the clinical application of NOTES is still more, but most of the abdominal approach is transvaginal, but it has gender specificity. There are few studies on transgastric gallbladder preservation, which may be related to the safety of postoperative gastric channel closure and complications of gastrointestinal closure. Moreover, the operation requirements, equipment, operation cost and time of NOTES are very high. Although NOTES have many advantages over laparoscopic or traditional open surgery, it could not be completely sure that NOTES surgery is better than laparoscopic surgery. Different characteristics of patients have the most appropriate treatment, and we can't totally deny which one. But the emergence of NOTES has also opened a milestone for the development of minimally invasive surgery. In the future, the development of NOTES is of great significance in surgery.

\section{Acknowledgements}

This work was supported in part by Program of Taizhou Science and Technology Grant (20ywb29, 1701KY22, 1701KY23), the Major Research Program of Taizhou Enze Medical Center Grant (19EZZDA2) and Foundation of "apricot grove scholar" of Chengdu University of Traditional Chinese Medicine (2019yky10).

\section{References}

1. Lammert F, Gurusamy K, Cynthia W Ko, Miquel JF, Sanchez NM, et al. (2016) Gallstones. Nat Rev Dis Primers 2: 16024.
2. Li Y, S Han (2020) Transgastric endoscopic gallbladder polypectomy and cholecystolithiasis: A case report. Exp Ther Med 19(1): 95-98.

3. Wang YL, Xia SH, Li HC, Gao F, Qi LN, et al. (2018) Transgastric natural orifice transluminal endoscopic gallbladder-preserving surgery: Report of three cases. World Chin J Digestol 26(23): 1423-1428.

4. Di Ciaula A, Garruti G, Q-H Wang D, Portincasa P (2018) Cholecystectomy and risk of metabolic syndrome. Eur J Intern Med 53: 3-11.

5. Lamberts MP (2018) Indications of cholecystectomy in gallstone disease. Curr Opin Gastroenterol 34(2): 97-102.

6. Zhu X, Liu J, Wang F, Zhao Q Zhang X, et al. (2020) Influence of traditional Chinese culture on the choice of patients concerning the technique for treatment of cholelithiasis: Cultural background and historical origins of gallbladder-preserving surgery. Surgery 167(2): 279-282.

7. Yip HC, PW y-Chiu (2015) Recent advances in natural orifice transluminal endoscopic surgery. Eur J Car Thor Sur 49(Suppl 1): i25-i30.

8. Liu L, WY Chiu P, Reddy N, Ho KY, Kitano S, et al. (2013) Natural orifice transluminal endoscopic surgery (NOTES) for clinical management of intra-abdominal diseases. Dig Endosc 25(6): 565-77.

9. Yoshiki N (2017) Review of transvaginal natural orifice transluminal endoscopic surgery in gynecology. Gynecol Minim Invasive Ther 6(1): $1-5$.

10. de Bari O, Wang TY, Liu M, Portincasa P, Q-H Wang D (2015) Estrogen induces two distinct cholesterol crystallization pathways by activating $\mathrm{ER} \alpha$ and GPR30 in female mice. J Lipid Res 56(9): 1691-700.

11. Salgaonkar H, R Parameswaran (2019) Adrenal natural orifice transluminal endoscopic surgery (NOTES): a step too far? Gland Surg 8(Suppl 1): S17-S21.

12. Zuo S, S Wang (2016) Current and emerging robotic assisted intervention for Notes. Expert Rev Med Devices 13(12): 1095-1105.

13. Fuchs KH (2015) Comments on the current status and future development of natural orifice transluminal endoscopic surgery. ANZ J Surg 85(4): 201-202.

14. Arezzo A, Zornig C, Mofid H, Fuchs KH, Breithaupt W, et al. (2013) The EURO-NOTES clinical registry for natural orifice transluminal endoscopic surgery: a 2-year activity report. Surg Endosc 27(9): 3073-84.

15. Arroyo Vazquez J, Bergstrom M, Dot J, Suboh-Abadia MA, Fonseca C, et al. (2016) Surgical Trauma Caused by Different Abdominal Access RoutesComparison of Open Surgical, Laparoscopic, and NOTES Transgastric Techniques in a Porcine Model. J Laparoendosc Adv Surg Tech A 26(7): 511-516.

16. Saxena P, MA Khashab (2016) New NOTES Clinical Training and Program Development. Gastrointest Endosc Clin N Am 26(2): 385-400.

17. Cai MY, PH Zhou, LQ Yao (2012) Current status of endoscopic resection in China. Dig Endosc 24 (Suppl 1): 166-71.

18. Cai MY, F Martin Carreras-Presas, PH Zhou (2018) Endoscopic fullthickness resection for gastrointestinal submucosal tumors. Dig Endosc 30 (Suppl 1): 17-24.

19. Liu Z, Wang Y, Fang Y, Huang Y, Yang H, et al. (2020) Short-term safety and efficacy of peroral endoscopic myotomy for the treatment of achalasia in children. J Gastroenterol 55(2): 159-168.

20. Qi ZP, Chen T, Li B, Ren Z, Yao LQ et al. (2018) Endoscopic submucosal dissection for early esophageal cancer in elderly patients with relative indications for endoscopic treatment. Endoscopy 50(9): 839-845.

21. Miakicheva O, Hamilton Z, Beksac AT, Berquist SW, Hassan AE, et al. (2016) Gastrointestinal tract access for urological natural orifice transluminal endoscopic surgery. World J Gastrointest Endosc 8(19): 684-689.

22. Bingener J, I Ibrahim-zada (2014) Natural orifice transluminal endoscopic surgery for intra-abdominal emergency conditions. Br J Surg 101(1): e80-e89. 
23. Erridge S, Sodergren MH, Darzi A, Purkayastha S (2016) Natural Orifice Translumenal Endoscopic Surgery: Review of Its Applications in Bariatric Procedures. Obes Surg 26(2): 422-428.

24. Al Talhi Y, Shirah BH, Altowairqi M, Yousef Y (2017) Laparoscopic cholecystectomy for cholelithiasis in children with sickle cell disease. Clin J Gastroenterol 10(4): 320-326.

25. Chen CH, CL Lin, CH Kao (2020) Association of Cholecystectomy with the Risk of Prostate Cancer in Patients with Gallstones. Cancers (Basel) 12(3): 544

26. Liu Y, He Y, Li T, Xie L, Wang J, et al. (2014) Risk of primary liver cancer associated with gallstones and cholecystectomy: a meta-analysis. PLoS One 9(10): e109733.

27. Su H, Huang L, Han CM, Lin YJ, Yen CF, et al. (2018) Natural orifice transluminal endoscopic surgery (NOTES) subtotal hysterectomy: A feasibility study. Taiwan J Obstet Gynecol 57(3): 355-359.

28. Khashab MA, AN Kalloo (2011) Critical analysis of hot topics in NOTES. Nat Rev Gastroenterol Hepatol 8(10): 565-572.

29. Atallah S, B Martin-Perez, D Keller, J Burke, L Hunter (2015) Naturalorifice transluminal endoscopic surgery. British Journal of Surgery 102(2): e73-e92.

30. Magdeburg R, G Kaehler (2016) Natural orifice transluminal endoscopic surgery in humans: feasibility and safety of transgastric closure using the OTSC system. Surg Endosc 30(1): 73-77.

31. Tarantino G, Magistri P, Ballarin R, Assirati G, Cataldo AD, et al. (2017) Surgery in biliary lithiasis: from the traditional "open" approach to

ISSN: 2574-1241

DOI: $10.26717 /$ BJSTR.2020.30.004936

Chi Liu, Shao-wei Li. Biomed J Sci \& Tech Res

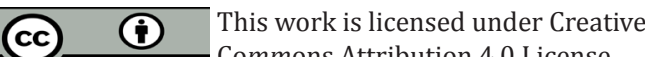

Submission Link: https://biomedres.us/submit-manuscript.php laparoscopy and the "rendezvous" technique. Hepatobiliary Pancreat Dis Int 16(6): 595-601.

32. Chen CH, CL Lin, CH Kao (2020) The Risk of Venous Thromboembolism in Patients with Gallstones. Int J Environ Res Public Health 17(8): 2930.

33. Metman MJH, Olthof PB, BC van der Wal J, M van Gulik TM, Roos D, et al. (2020) Clinical relevance of gallbladder polyps; is cholecystectomy always necessary? HPB (Oxford) 22(4): 506-510.

34. Ryou, M, Hazan R, Rahme L, Thompson CC (2012) An ex vivo bacteriologic study comparing antiseptic techniques for natural orifice translumenal endoscopic surgery (NOTES) via the gastrointestinal tract. Dig Dis Sci 57(8): 2130-2136.

35. Giday SA, Dray X, Magno P, Buscaglia JM, Shin EJ, et al. (2010) Infection during natural orifice transluminal endoscopic surgery: a randomized, controlled study in a live porcine model. Gastrointest Endosc 71(4): 812816.

36. Khashab MA, AN Kalloo (2010) Natural orifice translumenal endoscopic surgery. Curr Opin Gastroenterol 26(5): 471-477.

37. Fisichella PM, DeMeester SR, Hungness E, Perretta S, Soper NJ, et al. (2015) Emerging Techniques in Minimally Invasive Surgery. Pros and Cons. J Gastrointest Surg 19(7): 1355-1362.

38. Yang YS (2020) Robotic natural orifice transluminal endoscopic surgery (NOTES) hysterectomy as a scarless and gasless surgery. Surg Endosc 34(1): 492-500

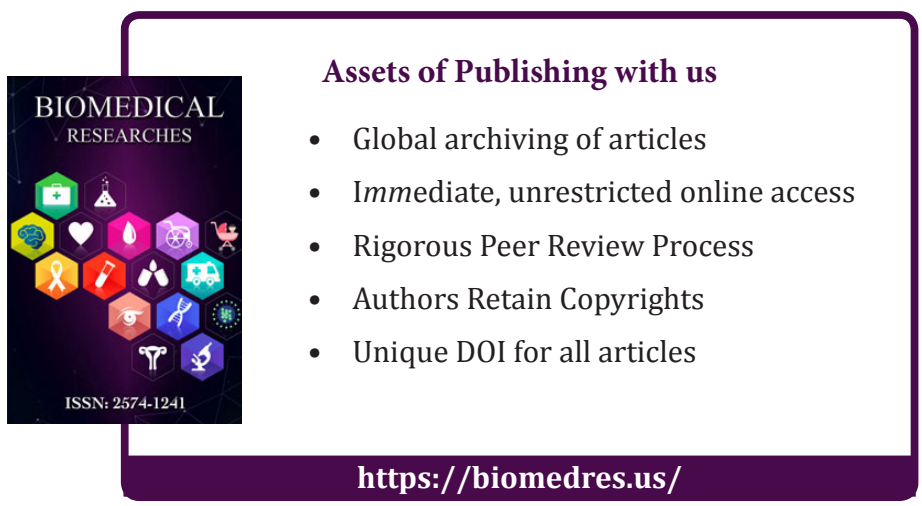

